\title{
The pure BRST Einstein-Hilbert Lagrangian from the double-copy to cubic order
}

\section{Borsten ${ }^{a, b}$ and S. Nagy ${ }^{c, 1}$}

${ }^{a}$ Maxwell Institute for Mathematical Sciences, Department of Mathematics, Heriot-Watt University, Colin Maclaurin Building, Riccarton, Edinburgh EH14 4AS, United Kingdom

${ }^{b}$ School of Theoretical Physics, Dublin Institute for Advanced Studies, 10 Burlington Road, Dublin 4, Ireland

${ }^{c}$ Centre for Astronomy \& Particle Theory, University Park, Nottingham, NG7 2RD, United Kingdom

E-mail: 1.borsten@hw.ac.uk, silvia.nagy@nottingham.ac.uk

ABSTRACT: We construct the pure gravity Becchi-Rouet-Stora-Tyutin (BRST) EinsteinHilbert Lagrangian, to cubic order, using the BRST convolution product of two Yang-Mills theories, in conjunction with the Bern-Carrasco-Johansson (BCJ) double-copy.

Keywords: BRST Quantization, Classical Theories of Gravity, Scattering Amplitudes ARXiv EPrint: 2004.14945

\footnotetext{
${ }^{1}$ Corresponding author.
} 


\section{Contents}

1 Introduction 1

2 A linearised tale told again (with ghosts) 3

2.1 Review of convolution dictionary and the necessity of the BRST framework 3

2.2 Dictionary and gauge mapping 4

$\begin{array}{lll}2.3 & \text { Pure gravity } & 6\end{array}$

3 Einstein-Hilbert from the double-copy to cubic order $\quad 8$

3.1 Gravity as it comes 8

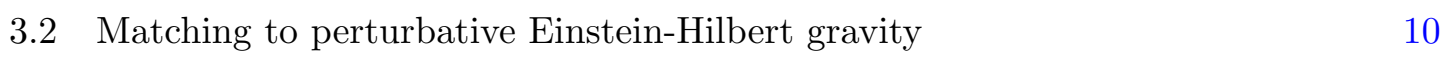

4 Conclusions 12

\section{Introduction}

In the present contribution we construct the pure gravity Lagrangian, to cubic order, using the BRST convolution product of two Yang-Mills theories introduced in $[1,2]$, in conjunction with the BCJ double-copy $[3-5]$ and, in particular, the developments of $[5,6]$.

The BCJ colour-kinematic duality conjecture [3-5] implies that the amplitudes of $\mathcal{N}=0$ supergravity,

$$
S_{\mathcal{N}=0}=\frac{1}{2 \kappa^{2}} \int \star R-\frac{1}{(D-2)} d \varphi \wedge \star d \varphi-\frac{1}{2} e^{-\frac{4}{D-2} \varphi} H \wedge \star H
$$

where $\varphi$ is the dilaton and $H=d B$ is the Kalb-Ramond (KR) 2-form field strength, follow from the double-copy of the amplitudes of pure Yang-Mills theory with arbitrary non-Abelian gauge group,

$$
S_{\mathrm{YM}}=\frac{1}{2 g^{2}} \operatorname{tr} \int F \wedge \star F,
$$

to all orders in perturbation theory.

Recall, $\mathcal{N}=0$ supergravity is the common NS-NS sector of the $\alpha^{\prime} \rightarrow 0$ limit of closed string theories. In this context, its appearance is understood to be a consequence of "open $\times$ open $=$ closed" property of the string spectrum underlying the Kawai-Lewellen-Tye (KLT) tree-level scattering amplitude relations of string theory [7]. At the level of the massless on-shell states we have the straighforward tensor product,

$$
A_{i} \otimes A_{j}=h_{i j} \oplus B_{i j} \oplus \varphi
$$

where $i, j=1, \ldots, D-2$. 
The BCJ duality for gluons has been established to all orders at tree-level from a number of perspectives [8-11] and has been generalised to include numerous (super) YangMills theories [4, 5, 12-38], generating a wide variety of double-copy constructible gravity theories. Although BCJ duality remains conjectural at loop-level, there is a growing list of highly non-trivial examples [4, 14, 29, 36, 39-55].

This programme is suggestive of a deep "gravity $=$ gauge $\times$ gauge" relation and has already dramatically advanced our understanding of perturbative quantum gravity. See for example $[12,43,52,53,55,56]$. This motivates an effort to understand the degree to which the paradigm can be pushed beyond scattering amplitudes and the identification of asymptotic on-shell states as in (1.3). For example, it has been shown that one can manifest BCJ duality at the level of the Lagrangian or field equations from a number of points of view [3, 4, 57-63]. A related line of research has been the construction of classical solutions in theories of gravity, such as black holes, from gauge theory solutions. This comes either in the form of applying a classical double-copy-type map to classical gauge theory solutions or extracting perturbative classical solutions from the double-copy of gauge theory amplitudes [6, 64-99]. This has yielded both pragmatic applications, particularly in the context of gravity-wave astronomy, as well as emphasising interesting questions and features of the double-copy itself. For a review of these ideas, including the many topics not mentioned here, their applications and further references see [100-102].

The approach taken here makes key use of the field theoretic convolutive product of gauge theories, introduced in [1] and further developed in [2, 28, 70-72, 74, 103, 104]. Using the product, the local symmetries and equations of motion of the resulting gravity theory have been shown to arise from those of the gauge theory factors, to linear order, making crucial use of the BRST formalism [2, 72, 104, 105]. The procedure was recently extended beyond Minkowski backgrounds in [105] and applied to the Janis-Newman-Winicour (JNW) solution in [72]. Given enough global symmetries in the gauge theory factors, this can then be used to identify the corresponding gravitational theory and all its symmetries uniquely $[1,28,32,70,71,103,104,106-110]$. The field theoretic product is a priori independent from the BCJ procedure, however it is consistent with it in the sense that the double-copy amplitudes correspond to the theory obtained from the field product, as seen from the matching of symmetries.

Another important and early development [5] particularly relevant here, promotes BCJ duality and the double-copy to the level of actions. First, it was shown that the YangMills actions may be put into a purely cubic form that manifestly yields colour-kinematic dual tree-level amplitudes by introducing an infinite tower of auxiliary fields [5]. This was demonstrated explicitly to five-points in [5] and a systematic formulation of the manifestly tree-level BCJ respecting action to all orders was given in [59], albeit without auxiliary fields so that it is necessarily non-local. Given Yang-Mills theory written in such a form, the double-copy principle can be straightforwardly applied to generate an action that correctly reproduces all the tree-level amplitudes of perturbative $\mathcal{N}=0$ supergravity, as described in [5] and developed in context of perturbative solutions in [6].

One of the main advantages of the BRST formulation of the field theory convolution product is that it gives an elegant solution to the issue of the mixing of the dilaton and 
graviton degrees of freedom pointed out in e.g. [6, 74], as detailed in $[2,72,102,105] .{ }^{1}$ In particular, the BRST approach opens the door to an off-shell construction for pure Einstein-Hilbert gravity. As such, it is desirable to extend the construction beyond linear order. To this end we employ the field theory convolution product and BRST prescription of $[2,105]$ in tandem with the double-copy applied to actions, as developed in $[5,6]$, to reproduce the BRST Einstein-Hilbert Lagrangian to cubic order.

The paper is organised as follows: we give an overview of the linearised BRST doublecopy procedure in section 2, applied to the simplest case of the product of two gauge fields. We demonstrate in particular how the ghost fields allow us to truncate to pure gravity in a robust way. We extend the construction to cubic order in perturbation theory in section 3, demonstrating that the BCJ construction applied to the BRST Yang-Mills action to cubic order reproduces Einstein-Hilbert gravity, up to field redefinitions. We also give an algorithm for mapping between the gauge-fixing functionals of the gauge theory and gravity sides. We conclude in section 4 .

\section{A linearised tale told again (with ghosts)}

\subsection{Review of convolution dictionary and the necessity of the BRST frame- work}

At linear level, the double copy dictionary is constructed from an associative convolutive inner tensor product with respect to the Poincaré group

$$
[f \cdot g](x)=\int d^{D} y f(y) \otimes g(x-y) .
$$

We will use the notation

$$
[f \circ \tilde{f}](x)=\left[f^{a} \cdot \Phi_{a \tilde{a}} \cdot \tilde{f}^{\tilde{a}}\right](x),
$$

where $\Phi$ is the convolutive pseudo-inverse $\Phi=\phi^{-1}$, with $\phi \cdot \Phi \cdot \phi=\phi$ of the bi-adjoint scalar $\phi$ of the BCJ zeroth-copy $[22,23,33,60,62,64,65,67,68,111-118]$. Note the circle product can be generalised to include fundamental matter fields, by including a bi-fundamental scalar field [28]. The product (2.2) applied to left $A_{\mu}$ and right $\tilde{A}_{\nu}$ pure Yang-Mills theories would be expected to yield $\mathcal{N}=0$ supergravity off-shell, given the BCJ amplitude relations and the tensor product of the on-shell states (1.3).

However, this expectation is only met once the BRST formalism is incorporated. This can be traced back to a number of issues that have been identified in relation to this construction in the context of off-shell or classical approaches:

- It is difficult to disentangle the graviton and dilaton degrees of freedom [74, 75]. A formal demonstration of this is presented in [74]. Let $j_{\mu}$ and $\tilde{j}_{\mu}$ be the sources of the Yang-Mills equation of motion, $j_{\mu \nu}^{(h)}$ the graviton source and $j^{(\varphi)}$ the dilaton source. Then we have

$$
j^{(\varphi)} \propto j_{\rho}^{(h) \rho} \propto \frac{1}{\square} j_{\rho} \circ \tilde{j}^{\rho} .
$$

\footnotetext{
${ }^{1}$ For related considerations in the context of the classical Kerr-Schild double-copy see [96].
} 
Thus we see that the graviton and dilaton sources are not independent. In particular, choosing to not source the dilaton will severely restrict the graviton. We can interpret this as a constraint on gravitational theories that admit a double copy description, appearing already at the linear order.

- The above comments are a general feature of the classical BCJ double-copy, and not a consequence of the set-up in [74]. This is evident in the mismatch between the on-shell and off-shell degrees of freedom. Specifically, $A_{\mu} \times \tilde{A}_{\nu}$ has $3 \times 3$ degrees of freedom off-shell, which is insufficient to describe the ten off-shell degrees of freedom carried by the graviton-two-form-dilaton system $[2,74]$. This issue only becomes more apparent with the addition of supersymmetry, where one lacks a full supermultiplets of off-shell degrees of freedom $[1,119,120]$.

- The classical double-copy is usually formulated with some specific gauge fixing on both the Yang-Mills and the gravity side. However, there is no general procedure determining a mapping between these corresponding gauge choices, potentially introducing ambiguities into the double-copy when taken beyond the domain of amplitudes.

The BRST dictionary in [2] resolves the above issues by taking products of sets of fields $\left(A_{\mu}, c^{\alpha}\right)$ and $\left(\tilde{A}_{\mu}, \tilde{c}^{\alpha}\right)$. Here $c^{1}=c$ and $c^{2}=\bar{c}$ are the Fadeev-Popov ghost and antighost, respectively. The off-shell d.o.f. of the $\left(A_{\mu}, c^{\alpha}\right) \times\left(\tilde{A}_{\mu}, \tilde{c}^{\alpha}\right)$ product can now be seen to correspond to those of the linearised BRST systems for the graviton, two-form and dilaton. ${ }^{2}$ It also naturally incorporates the ghost and ghost-for-ghost transformations [2, 121].

We will describe in section 2.3 how the BRST procedure resolves the source issue (2.3), and allows us to obtain a pure gravity theory. We will also present the gauge mapping algorithm between pure Yang-Mills theory and gravity coupled to a KR 2-form and a dilaton in section 2.2 .

\subsection{Dictionary and gauge mapping}

The general form of the BRST action is (having eliminated the Nakanishi-Lautrup auxiliary field), schematically:

$$
S_{\mathrm{BRST}}=\int d^{D} x\left(\mathcal{L}_{0}[f]+\frac{1}{2 \xi} G[f]^{2}-\bar{c} Q(G[f])\right)-f j^{(f)}+\bar{j} c+\bar{c} j,
$$

where $\mathcal{L}_{0}[f]$ is the classical action for the field $f, G[f]$ is the gauge-fixing functional and $\bar{c} Q G[f]$ is the ghost Lagrangian. For reducible gauge symmetries there will be additional ghost-for-ghost terms. For a review of the BRST procedure, see [122-125]. The left and right (tilde) Yang-Mills fields and ghosts transform as:

$$
\begin{aligned}
Q A_{\mu}=\partial_{\mu} c, & Q c=0, & Q \bar{c}=\frac{1}{\xi} G(A), \\
Q \tilde{A}_{\mu}=\partial_{\mu} \tilde{c}, & Q \tilde{c}=0, & Q \overline{\tilde{c}}=\frac{1}{\tilde{\xi}} G(\tilde{A}),
\end{aligned}
$$

\footnotetext{
${ }^{2}$ Note that the d.o.f. counting is now graded by ghost number — see [2] for details.
} 
while those of linearised $\mathcal{N}=0$ supergravity transform as:

$$
\begin{aligned}
& Q h_{\mu \nu}=2 \partial_{(\mu} c_{\nu)}, \quad Q c_{\mu}=0, \quad Q \bar{c}_{\mu}=\frac{1}{\xi^{(h)}} G_{\mu}^{(h)}, \\
& Q B_{\mu \nu}=2 \partial_{[\mu} d_{\nu]}, \quad Q d_{\mu}=\partial_{\mu} d, \quad Q \bar{d}_{\mu}=\frac{1}{\xi^{(B)}} G_{\mu}^{(B)}, \\
& Q \varphi=0 .
\end{aligned}
$$

The BRST system for the two-form additionally contains the (anti)ghost-for-ghosts $\bar{d}, d$ and the ghost number 0 object $\eta$, transforming as

$$
Q d=0, \quad Q \bar{d}=\frac{1}{\xi_{(d)}} \partial^{\mu} \bar{d}_{\mu}, \quad Q \eta=\frac{m_{(d)}}{\xi_{(d)}} \partial^{\mu} d_{\mu}
$$

with $\xi_{(d)}$ and $m_{(d)}$ some a priori arbitrary constants. It is convenient to make a choice of gauge fixing functional on the Yang-Mills side, and set

$$
G[A] \equiv \partial^{\mu} A_{\mu}, \quad G[\tilde{A}] \equiv \partial^{\mu} \tilde{A}_{\mu} .
$$

As derived in $[2,105]$ a simple dictionary for the linearised fields of $\mathcal{N}=0$ supergravity compatible with the symmetries above is given by

$$
\begin{aligned}
h_{\mu \nu} & =A_{\mu} \circ \tilde{A}_{\nu}+A_{\nu} \circ \tilde{A}_{\mu}+a \eta_{\mu \nu}\left(A^{\rho} \circ \tilde{A}_{\rho}+\tilde{\xi} c \circ \tilde{\bar{c}}-\xi \bar{c} \circ \tilde{c}\right), \\
B_{\mu \nu} & =A_{\mu} \circ \tilde{A}_{\nu}-A_{\nu} \circ \tilde{A}_{\mu}, \\
\varphi & =A^{\rho} \circ \tilde{A}_{\rho}+\tilde{\xi} c \circ \tilde{\bar{c}}-\xi \bar{c} \circ \tilde{c},
\end{aligned}
$$

where $a$ is an arbitrary parameter. We can immediately read off the graviton and two-form ghost dictionaries,

$$
\begin{aligned}
& c_{\mu}=c \circ \tilde{A_{\mu}}+A_{\mu} \circ \tilde{c}, \\
& d_{\mu}=c \circ \tilde{A_{\mu}}-A_{\mu} \circ \tilde{c},
\end{aligned}
$$

from which the antighost dictionaries follow:

$$
\begin{aligned}
& \bar{c}_{\mu}=\bar{c} \circ \tilde{A}_{\mu}+A_{\mu} \circ \tilde{\bar{c}}, \\
& \bar{d}_{\mu}=\bar{c} \circ \tilde{A_{\mu}}-A_{\mu} \circ \tilde{\bar{c}} .
\end{aligned}
$$

Finally, the second order ghosts in the Kalb-Ramond sector are given by

$$
d=-2 c \circ \tilde{c}, \quad \bar{d}=-2 \bar{c} \circ \tilde{\bar{c}}, \quad \eta=-(\tilde{\xi} c \circ \tilde{\bar{c}}+\xi \bar{c} \circ \tilde{c})
$$

A significant advantage of the BRST set-up is that we can directly derive the gaugefixing functional for the graviton and the two-form, given the Yang-Mills gauge-fixing functional. Indeed, using (2.11) and (2.6), in conjunction with the Yang-Mills transformations (2.5), we can determine the graviton and two-form gauge-fixing functionals through

$$
Q \bar{c}_{\mu}=\frac{1}{\xi^{(h)}} G_{\mu}^{(h)}, \quad Q \bar{d}_{\mu}=\frac{1}{\xi^{(B)}} G_{\mu}^{(B)}
$$


and, making use of the Yang-Mills transformations (2.5), we get

$$
\begin{aligned}
G_{\mu}^{(h)} & =\frac{\xi^{(h)}(\tilde{\xi}+\xi)}{2 \xi \tilde{\xi}}\left[\partial^{\nu} h_{\nu \mu}-\frac{1}{2} \partial_{\mu} h+\frac{2+(D-2) a}{2} \partial_{\mu} \varphi\right]+\frac{\xi^{(h)}(\tilde{\xi}-\xi)}{2 \xi \tilde{\xi}}\left[\partial^{\nu} B_{\nu \mu}+\partial_{\mu} \eta\right], \\
G_{\mu}^{(B)} & =\frac{\xi^{(B)}(\tilde{\xi}+\xi)}{2 \xi \tilde{\xi}}\left[\partial^{\nu} B_{\nu \mu}+\partial_{\mu} \eta\right]+\frac{\xi^{(B)}(\tilde{\xi}-\xi)}{2 \xi \tilde{\xi}}\left[\partial^{\nu} h_{\nu \mu}-\frac{1}{2} \partial_{\mu} h+\frac{2+(D-2) a}{2} \partial_{\mu} \varphi\right] .
\end{aligned}
$$

Note, one can repackage these gauge condition into left/right transverse gauges for the trace-reversed generalised metric $\bar{Z}_{\mu \nu}=\bar{h}_{\mu \nu}+B_{\mu \nu}$,

$$
\begin{aligned}
& G_{\mu}^{(h)}=\frac{1}{2 \xi}\left(\partial^{\rho} \bar{Z}_{\rho \mu}+\partial_{\mu} \chi^{+}\right)+\frac{1}{2 \tilde{\xi}}\left(\partial^{\rho} \bar{Z}_{\mu \rho}+\partial_{\mu} \chi^{-}\right), \\
& G_{\mu}^{(B)}=\frac{1}{2 \xi}\left(\partial^{\rho} \bar{Z}_{\rho \mu}+\partial_{\mu} \chi^{+}\right)-\frac{1}{2 \tilde{\xi}}\left(\partial^{\rho} \bar{Z}_{\mu \rho}+\partial_{\mu} \chi^{-}\right),
\end{aligned}
$$

where

$$
\chi^{ \pm}=\frac{(2+D a)}{2} \varphi \pm \eta .
$$

Here we see the rôle of the dilaton appearing in the gauge-fixing functional in direct analogy to the familiar appearance of $\eta$ in the KR gauge-fixing functional. This reflects the fact that it receives a contribution from the ghost-antighost sector of Yang-Mills squared, $\tilde{\xi} c \circ \tilde{\bar{c}}-\xi \bar{c} \circ \tilde{c}$.

For $\xi=\tilde{\xi}=\xi^{(h)}=\xi^{(B)}$ and $a=\frac{2}{2-D}$, the gauge fixing functionals reduce to

$$
\begin{aligned}
G_{\mu}^{(h)} & =\partial^{\nu} h_{\nu \mu}-\frac{1}{2} \partial_{\mu} h, \\
G_{\mu}^{(B)} & =\partial^{\nu} B_{\nu \mu}+\partial_{\mu} \eta,
\end{aligned}
$$

the natural choices for Einstein frame.

Knowledge of the gauge fixing functionals (2.17) now allows us to write the linearised Lagrangians:

$$
\begin{aligned}
\mathcal{L}(h, \varphi) & =\frac{1}{4} h^{\mu \nu} \square h_{\mu \nu}+\frac{1+\xi}{2 \xi}\left(\partial^{\mu} h_{\mu \rho} \partial_{\nu} h^{\nu \rho}-\partial^{\mu} h \partial^{\nu} h_{\mu \nu}\right)-\frac{1+2 \xi}{8 \xi} h \square h-\bar{c}^{\mu} \square c_{\mu}+\frac{1}{2} \varphi \square \varphi \\
\mathcal{L}(B) & =\frac{1}{4} B^{\mu \nu} \square B_{\mu \nu}+\frac{1+\xi}{2 \xi} \partial^{\mu} B_{\mu \nu} \partial_{\rho} B^{\rho \nu}-\bar{d}^{\mu} \square d_{\mu}+\frac{\xi}{2} \bar{d} \square d-\frac{1}{2 \xi} \eta \square \eta .
\end{aligned}
$$

\subsection{Pure gravity}

Here we remove the dilaton and KR two-form to leave pure Einstein-Hilbert gravity. First, it is straightforward to see from (2.9), (2.10), (2.11) and (2.12) that we can truncate out the entire Kalb-Ramond sector by identifying the two Yang-Mills theories: ${ }^{3}$

$$
A_{\mu}=\tilde{A}_{\mu}, \quad c=\tilde{c}, \quad \bar{c}=\tilde{\bar{c}} .
$$

\footnotetext{
${ }^{3}$ Remember that $A_{\mu} \circ \tilde{A}_{\nu}=A_{\mu}^{a} \cdot \Phi_{a \tilde{a}} \cdot \tilde{A}_{\nu}^{\tilde{a}}$, where we are summing over the adjoint indices $a, \tilde{a}$. In principle, this would allow us to more generally set the two-form sector to vanish without picking $\left(A_{\mu}, c, \bar{c}\right)=$ $\alpha\left(\tilde{A}_{\mu}, c, \bar{c}\right)$, with $\alpha$ some constant. However, we find it convenient to make this choice, and set $\alpha=1$.
} 
To illustrate how the dilaton can be removed, we couple arbitrary sources to the right hand side of the Yang-Mills eom:

$$
\square A_{\mu}-\frac{\xi+1}{\xi} \partial_{\mu} \partial A=j_{\mu}, \quad \square c=j, \quad \square \bar{c}=\bar{j} .
$$

Note that, in contrast with the standard treatment of BRST, we have coupled sources to the ghost/antighost. The graviton/dilaton equations, as coming from (2.18) coupled to sources, become:

$$
\begin{aligned}
\frac{1}{2} \square h_{\mu \nu}-\frac{1+\xi}{\xi} \partial^{\rho} \partial_{(\mu} h_{\nu) \rho}+\frac{1+\xi}{2 \xi} \partial_{\mu} \partial_{\nu} h+\eta_{\mu \nu}\left[\frac{1+\xi}{2 \xi} \partial^{\rho} \partial^{\sigma} h_{\rho \sigma}-\frac{1+2 \xi}{4 \xi} \square h\right] & =j_{\mu \nu}^{(h)} \\
\square \varphi & =j^{(\varphi)}
\end{aligned}
$$

Then using (2.20) and (2.9), we can read off the source dictionaries,

$$
\begin{aligned}
j_{\mu \nu}^{(h)}= & \frac{1}{\square} j_{\mu} \circ j_{\nu}-\frac{2(1+\xi)}{\square^{2}} \partial_{\mu} \partial_{\nu} j \circ \bar{j}-\frac{(\xi+1)^{2}}{\square^{3}} \partial_{\mu} \partial_{\nu} \partial j \circ \partial j \\
& +\eta_{\mu \nu}\left[\frac{\xi(1+\xi)}{\square^{2}} \partial j \circ \partial j+\frac{1+2 \xi}{\square} j \circ \bar{j}\right] \\
j^{(\varphi)}= & \frac{1}{\square} j^{\rho} \circ j_{\rho}+\frac{\xi^{2}-1}{\square^{2}} \partial j \circ \partial j+\frac{2 \xi}{\square} j \circ \bar{j},
\end{aligned}
$$

where from here-on-in we set $D=4$ for notational clarity, although all of the conclusions hold for arbitrary dimension. If we wish to eliminate the dilaton, we first set its source to vanish by picking sources for the ghosts such that

$$
j \circ \bar{j}=-\frac{1}{2 \xi} j^{\rho} \circ j_{\rho}-\frac{\xi^{2}-1}{2 \xi} \frac{1}{\square} \partial j \circ \partial j
$$

which allows us to set

$$
c \circ \bar{c}=-\frac{1}{2 \xi} A^{\rho} \circ A_{\rho} \quad \Rightarrow \quad \varphi=0 .
$$

The graviton source reduces to

$$
\begin{aligned}
j_{\mu \nu}^{(0)}= & \frac{1}{\square} j_{\mu} \circ j_{\nu}+\frac{1+\xi}{\xi \square^{2}} \partial_{\mu} \partial_{\nu} j^{\rho} \circ j_{\rho}-\frac{(\xi+1)^{2}}{\xi \square^{3}} \partial_{\mu} \partial_{\nu} \partial j \circ \partial j \\
& -\eta_{\mu \nu}\left[\frac{1+2 \xi}{2 \xi \square} j^{\rho} \circ j_{\rho}-\frac{(\xi+1)^{2}}{2 \xi \square^{2}} \partial j \circ \partial j\right]
\end{aligned}
$$

and we note that it is unconstrained, even after eliminating the dilaton. On the other hand, in the absence of the ghost contributions setting the dilaton and its source to vanish would constrain the trace of (2.25) to be vanishing, cf. (2.3).

Finally, we can invert the dictionaries (2.9), (2.10) and (2.11) to get:

$$
\begin{aligned}
A_{\mu} \circ A_{\nu} & =\frac{1}{2} h_{\mu \nu} \\
c \circ A_{\mu} & =\frac{1}{2} c_{\mu} \\
\bar{c} \circ A_{\mu} & =\frac{1}{2} \bar{c}_{\mu} \\
c \circ \bar{c} & =-\frac{1}{4 \xi} h
\end{aligned}
$$


and the gauge-fixing functional reduces to the familiar de Donder gauge,

$$
G_{\mu}[h]=\partial^{\nu} h_{\nu \mu}-\frac{1}{2} \partial_{\mu} h
$$

with the pure gravity BRST action

$$
\mathcal{L}(h)=\frac{1}{4} h^{\mu \nu} \square h_{\mu \nu}+\frac{1+\xi}{2 \xi}\left(\partial^{\mu} h_{\mu \rho} \partial_{\nu} h^{\nu \rho}-\partial^{\mu} h \partial^{\nu} h_{\mu \nu}\right)-\frac{1+2 \xi}{8 \xi} h \square h-\bar{c}^{\mu} \square c_{\mu} .
$$

\section{Einstein-Hilbert from the double-copy to cubic order}

\subsection{Gravity as it comes}

We work with the standard Yang-Mills BRST action

$$
\mathcal{L}_{\mathrm{YM}}=-\frac{1}{4} F_{\mu \nu}^{a} F^{\mu \nu a}+\frac{1}{2 \xi} G[A]^{a} G[A]^{a}-\bar{c}^{a} \partial^{\mu} D_{\mu}^{a c} c^{c},
$$

where $F_{\mu \nu}^{a}=\partial_{\mu} A_{\nu}^{a}-\partial_{\nu} A_{\mu}^{a}+g f^{a b c} A_{\mu}^{b} A_{\nu}^{c}, D_{\mu}^{a c}=\delta^{a c} \partial_{\mu}+g f^{a b c} A_{\mu}^{b}$ and the gauge-fixing functional is linear $G[A]^{a}=\partial^{\rho} A_{\rho}^{a}$. Note, here Feynman gauge corresponds to $\xi=-1$. Up to cubic order this becomes

$$
\begin{aligned}
\mathcal{L}_{\mathrm{YM}}= & -\frac{1}{4} F_{\mu \nu}^{a(0)} F^{\mu \nu a(0)}+\frac{1}{2 \xi} \partial^{\rho} A_{\rho}^{a} \partial^{\rho} A_{\rho}^{a}-\bar{c}^{a} \square c^{a} \\
& -g f^{a b c} \partial_{\mu} A_{\nu}^{a} A^{\mu b} A^{\nu c}-g f^{a b c} \bar{c}^{a} \partial^{\mu}\left(A_{\mu}^{b} c^{c}\right),
\end{aligned}
$$

with $F^{(0)}=\partial_{\mu} A_{\nu}^{a}-\partial_{\nu} A_{\mu}^{a}$. The cubic terms can be written as

$$
\begin{aligned}
\mathcal{L}_{\mathrm{YM}}^{(3)}=i g f^{a b c} \int d p_{1} d p_{2} d p_{3} e^{-i\left(p_{1}+p_{2}+p_{3}\right) x} & {\left[\frac{1}{6} n^{\mu \nu \rho}\left(p_{i}\right) A_{\mu}^{a}\left(p_{1}\right) A_{\nu}^{b}\left(p_{2}\right) A_{\rho}^{c}\left(p_{3}\right)\right.} \\
& \left.+n^{\mu \alpha \beta}\left(p_{1}\right) c_{\alpha}^{a}\left(p_{1}\right) A_{\mu}^{b}\left(p_{2}\right) c_{\beta}^{c}\left(p_{3}\right)\right],
\end{aligned}
$$

with $d p=\frac{d^{4} p}{(2 \pi)^{4}}$ and we have isolated the BCJ satisfying, in the sense that it is totally antisymmetric, kinematic numerator

$$
n^{\mu_{1} \mu_{2} \mu_{3}}\left(p_{i}\right)=-\left(p_{12}^{\mu_{3}} \eta^{\mu_{1} \mu_{2}}+p_{23}^{\mu_{1}} \eta^{\mu_{2} \mu_{3}}+p_{31}^{\mu_{2}} \eta^{\mu_{3} \mu_{1}}\right)
$$

where $p_{i j}=p_{i}-p_{j}$. The above is as in classical Yang-Mills, ${ }^{4}$ however note that we now have a contribution coming from the ghost-antighost-gluon interaction term with kinetic numerator

$$
n^{\mu \alpha \beta}(p)=-p^{\mu} \sigma_{+}^{\alpha \beta}, \quad \sigma_{ \pm}=\frac{1}{2}\left(\sigma_{x} \pm i \sigma_{y}\right),
$$

where for convenience we have introduced the ghost-antighost doublet, $c_{\alpha}=(c, \bar{c})$. Here $\sigma_{i}$ are the Pauli matrices and $\sigma_{+}^{\alpha \beta} c_{\alpha} c_{\beta}$ creates a ghost number zero state.

\footnotetext{
${ }^{4}$ This is a consequence of choosing a linear gauge-fixing functional. It would be interesting to study models where $G$ is a nonlinear function of $A_{\mu}$.
} 
When performing the double-copy, we must take all possible combinations: the gluongluon-gluon term with itself will contribute graviton-graviton-graviton interactions, same as the ghost-antighost-gluon term with itself, while the cross terms will contribute the graviton-ghost-antighost interactions. Note, we have no a priori reason to believe the ghost sector of gravity generated through the naïve double-copy presented above will be consistent. However, as we shall demonstrate, it yields up to field redefinitions EinsteinHilbert gravity with BRST respecting gauge-fixing and ghost sectors, as required.

To implement the BCJ double-copy we introduce a super-index $M=(\mu, \alpha)$ and send $i f^{a b c} X^{M N P} \rightarrow \alpha_{(M N P, \tilde{M} \tilde{N} \tilde{P})} X^{\tilde{M} \tilde{N} \tilde{P}} X^{M N P}$, where there is no sum between the set of normalisation parameters, $\alpha$, and the non-zero components of $X$, which are determined by the allowed diagrams,

$$
X^{\mu \nu \rho}=n^{\mu \nu \rho}, \quad X^{\mu \alpha \beta}=n^{\mu \alpha \beta}, \quad X=0 \text { otherwise. }
$$

This yields the momentum space double-copy Lagrangian,

$$
\begin{aligned}
\hat{\mathcal{L}}_{\text {(grav) }}^{(3, d c)}= & \alpha_{(M N P, \tilde{M} \tilde{N} \tilde{P})} X^{\tilde{M} \tilde{N} \tilde{P}}\left[\frac{1}{6} n^{\mu \nu \rho} A_{\mu \tilde{M}} A_{\nu \tilde{N}} A_{\rho \tilde{P}}+n^{\nu \alpha \beta} c_{\alpha \tilde{M}} A_{\nu \tilde{N}} c_{\beta \tilde{P}}\right] \\
= & \frac{1}{6} \alpha_{1} n^{\mu \nu \rho} n^{\tilde{\mu} \tilde{\nu} \tilde{\rho}} A_{\mu \tilde{\mu}}(p) A_{\nu \tilde{\nu}}(k) A_{\rho \tilde{\rho}}(q)+\alpha_{3} p^{\mu} p^{\tilde{\mu}} \bar{C}^{(0)}(p) A_{\mu \tilde{\mu}}(k) C^{(0)}(q) \\
& -2 \alpha_{2}^{+} n^{\mu \nu \rho} p^{\tilde{\nu}} \bar{C}_{\mu}(p) A_{\nu \tilde{\nu}}(k) C_{\rho}(q),
\end{aligned}
$$

where for convenience we have labelled the non-zero constants ${ }^{5}$

$$
\alpha_{1}=\alpha_{(\mu \nu \rho, \tilde{\mu} \tilde{\nu} \tilde{\rho})}, \quad \alpha_{2}^{+}=\frac{1}{2}\left(\frac{1}{6} \alpha_{(\mu \nu \rho, \tilde{\mu} \tilde{\alpha} \tilde{\beta})}+\alpha_{(\mu \alpha \beta, \tilde{\mu} \tilde{\nu} \tilde{\rho})}\right), \quad \alpha_{3}=\alpha_{(\mu \alpha \beta, \tilde{\mu} \tilde{\alpha} \tilde{\beta})}
$$

and defined

$$
\begin{aligned}
A_{\mu \nu} & =\mathcal{F}\left[A_{\mu} \circ A_{\nu}\right] \\
C_{\mu} & =\mathcal{F}\left[A_{\mu} \circ c\right], \quad \bar{C}_{\mu}=\mathcal{F}\left[A_{\mu} \circ \bar{c}\right] \\
C^{(0)} & =\mathcal{F}[c \circ \bar{c}]=-\bar{C}^{(0)}
\end{aligned}
$$

with $\mathcal{F}$ denoting the Fourier transform. Making use of the linear dictionary (2.26), the graviton sector of the above can be rewritten in position space as

$$
\begin{aligned}
\mathcal{L}_{\text {(grav) }}^{(3, d c)}=-\frac{\alpha_{1}}{8} h^{\mu \nu}( & h^{\rho \sigma} \partial_{\rho} \partial_{\sigma} h_{\mu \nu}-\partial_{\mu} h^{\rho \sigma} \partial_{\nu} h_{\rho \sigma}-h^{\rho \sigma} \partial_{\sigma} \partial_{\nu} h_{\mu \rho} \\
& \left.+2 \partial_{\nu} h_{\rho \sigma} \partial^{\sigma} h_{\mu}{ }^{\rho}-\partial_{\rho} h_{\nu \sigma} \partial^{\sigma} h_{\mu}{ }^{\rho}-\frac{\alpha_{3}}{4 \xi^{2} \alpha_{1}} h \partial_{\mu} \partial_{\nu} h\right) .
\end{aligned}
$$

\footnotetext{
${ }^{5}$ Note that only a linear combination of the normalisation parameters $\alpha_{(\mu \nu \rho, \tilde{\mu} \tilde{\alpha} \tilde{\beta})}$ and $\alpha_{(\mu \alpha \beta, \tilde{\mu} \tilde{\nu} \tilde{\rho})}$ will be fixed through the double copy. This is a consequence of the fact that we have restricted to the symmetric sector in order to focus on pure gravity. In the full construction, the orthogonal combination

$$
\alpha_{2}^{-}=\frac{1}{2}\left(\frac{1}{6} \alpha_{(\mu \nu \rho, \tilde{\mu} \tilde{\alpha} \tilde{\beta})}-\alpha_{(\mu \alpha \beta, \tilde{\mu} \tilde{\nu} \tilde{\rho})}\right)
$$

will be fixed by studying the ghost sector of the two-form $B_{\mu \nu}$ [126].
} 
Note that this is simpler than the Einstein-Hilbert action at cubic order given in (3.12), thus revealing one of the advantages of the double copy dictionary. As observed in [5], the double-copy of the purely gluonic sector of the Yang-Mills action performed in this manner will give a graviton action that correctly reproduces the on-shell amplitudes to this order. The terms of (3.12) that vanish in the on-shell amplitude, due to the transverse-traceless polarisation tensors and momentum conservation, simply do not appear here. However, the ghost-antighost sector reintroduces a term depending on $h$, which allows one to fix the Einstein-frame dilaton to vanish, as will be demonstrated in section 3.2. Said another way, the vanishing of the Einstein-frame dilaton at linear order given in (2.24) remains consistent at higher orders.

\subsection{Matching to perturbative Einstein-Hilbert gravity}

At cubic level, the standard BRST action for gravity is

$$
\mathcal{L}^{3, B R S T}=\mathcal{L}_{\text {class }}^{(3)}+\frac{1}{\xi} G_{\mu}[h]^{(1)} G^{\mu}[h]^{(2)}-\left\{\bar{c}^{\mu} Q\left[G_{\mu}[h]\right]\right\}^{(3)},
$$

with the superscripts denoting the order in perturbation theory. $\mathcal{L}_{\text {class }}^{(3)}$ is just the cubic part of the Einstein-Hilbert action:

$$
\begin{aligned}
\mathcal{L}_{\text {class }}^{(3)}=\frac{1}{2} h^{\mu \nu} & \left(\frac{1}{2} \partial_{\mu} h^{\rho \sigma} \partial_{\nu} h_{\rho \sigma}-\frac{1}{4} \eta_{\mu \nu} \partial_{\sigma} h_{\tau \rho} \partial^{\sigma} h^{\tau \rho}+\partial_{\nu} h\left(\partial_{\rho} h_{\mu}{ }^{\rho}-\frac{1}{2} \partial_{\mu} h\right)\right. \\
& +\partial_{\nu} h_{\mu}{ }^{\rho} \partial_{\rho} h-\partial_{\rho} h \partial^{\rho} h_{\mu \nu}-\frac{1}{2} \eta_{\mu \nu} \partial^{\rho} h\left(\partial_{\sigma} h_{\rho}{ }^{\sigma}-\frac{1}{2} \partial_{\rho} h\right)+\partial^{\rho} h_{\mu \nu} \partial_{\sigma} h_{\rho}{ }^{\sigma} \\
& \left.-2 \partial_{\nu} h_{\rho \sigma} \partial^{\sigma} h_{\mu}{ }^{\rho}-\partial_{\rho} h_{\nu \sigma} \partial^{\sigma} h_{\mu}{ }^{\rho}+\partial_{\sigma} h_{\nu \rho} \partial^{\sigma} h_{\mu}{ }^{\rho}+\frac{1}{2} \eta_{\mu \nu} \partial_{\rho} h_{\tau \sigma} \partial^{\sigma} h^{\tau \rho}\right)
\end{aligned}
$$

The linear part of the gauge fixing functional is determined via the BRST procedure in $(2.27)$ :

$$
G_{\mu}[h]^{(1)}=\left[\partial^{\nu} h_{\nu \mu}-\frac{1}{2} \partial_{\mu} h\right],
$$

while the second order part $G_{\mu}[h]^{(2)}$ is to be determined by matching with the BCJ action (3.10). The normalisation factors in the double copy dictionary are fixed to

$$
\alpha_{1}=1, \quad \alpha_{3}=\xi^{2}
$$

and we find that one needs to perform a nonlinear field redefinition of the graviton fluctuation

$$
h_{\mu \nu} \rightarrow h_{\mu \nu}-\frac{1}{4} h_{\mu \nu} h+\frac{1}{2} h_{\mu}^{\rho} h_{\nu \rho}-\frac{1}{16} \eta_{\mu \nu}\left(h_{\rho \sigma} h^{\rho \sigma}-\frac{3}{4} h^{2}\right) .
$$

Comparing (3.11) with (3.10), we derive the next order in the gauge fixing functional prior to the field redefintion

$$
\begin{aligned}
G_{\mu}[h]^{(2)}= & \frac{3}{8} h^{\nu \rho} \partial_{\mu} h_{\nu \rho}-\frac{5+2 \xi}{32} h \partial_{\mu} h+\frac{4-3 \xi}{16} h_{\mu}{ }^{\rho} \partial_{\rho} h \\
& -\frac{1}{2} h^{\nu \rho} \partial_{\rho} h_{\mu \nu}+\frac{1}{4} h \partial^{\rho} h_{\mu \rho}-\frac{4-\xi}{4} h_{\mu}{ }^{\nu} \partial^{\rho} h_{\nu \rho} .
\end{aligned}
$$


Once the field redefinition (3.15) is applied, the gauge-fixing functional simplifies and is proportional to the free parameter $\xi$, as expected,

$$
G_{\mu}[h]^{(2)} \rightarrow \frac{\xi}{8}\left(h_{\mu}{ }^{\nu} \partial_{\rho} h_{\nu}{ }^{\rho}-\frac{1}{2} h \partial_{\mu} h-\frac{3}{2} h_{\mu}{ }^{\nu} \partial_{\nu} h\right) .
$$

Note, restricting to local field redefinitions, (3.15) and (3.17) are uniquely determined. Note moreover, there is no local field redefinition matching Einstein-Hilbert without the ghost-antighost sector. ${ }^{6}$

Once the gauge fixing term at second order has been found, the ghost terms in the cubic action are uniquely determined by the last term in (3.11), together with the perturbative BRST transformation

$$
Q h_{\mu \nu}=2 \partial_{(\mu} c_{\nu)}+\kappa\left[c^{\rho} \partial_{\rho} h_{\mu \nu}-2 c^{\rho} \partial_{(\mu} h_{\nu) \rho}\right]
$$

This can be matched to the BCJ ghost terms

$$
\begin{aligned}
\mathcal{L}_{(g h)}^{(3, d c)}=-\frac{\alpha_{2}^{+}}{4} & \left(h_{\nu \rho} \partial^{\rho} \bar{c}_{\mu} \partial^{\nu} c^{\mu}-h_{\mu \rho} \partial^{\rho} \bar{c}_{\nu} \partial^{\nu} c^{\mu}-\partial_{\mu} h_{\nu \rho} \partial^{\rho} \bar{c}^{\nu} c^{\mu}\right. \\
& \left.+\partial_{\nu} h_{\mu \rho} \partial^{\rho} \bar{c}^{\nu} c^{\mu}+h_{\nu \rho} \partial^{\rho} \partial_{\mu} \bar{c}^{\nu} c^{\mu}-h_{\nu \rho} \partial^{\rho} \partial^{\nu} \bar{c}_{\mu} c^{\mu}\right)
\end{aligned}
$$

by fixing the normalisation parameter

$$
\alpha_{2}^{+}=1
$$

and performing a non-local transformation on the ghost and antighost fields, which is not unique. A convenient, in the sense that it places no restrictions on the range of $\xi$, example is given by:

$$
\begin{aligned}
c_{\mu} \rightarrow & c_{\mu}+\frac{3 \xi-4}{32} h c_{\mu}+\frac{3}{4} h_{\mu}{ }^{\nu} c_{\nu}+\frac{1}{2 \square}\left[\frac{\xi+3}{8} \partial_{\rho} h \partial_{\mu} c^{\rho}+\frac{1}{2} \partial_{\mu} h \partial_{\rho} c^{\rho}\right. \\
& \left.-\frac{1}{2} \partial^{\sigma} h_{\mu \sigma} \partial^{\rho} c_{\rho}-\frac{\xi+2}{4} \partial^{\rho} h_{\sigma \rho} \partial_{\mu} c^{\sigma}-\frac{\xi+2}{4} \partial^{\rho} h_{\sigma \rho} \partial^{\sigma} c_{\mu}+\frac{\xi+1}{2} h_{\mu \rho} \partial^{\rho} \partial^{\sigma} c_{\sigma}\right] \\
\bar{c}_{\mu} \rightarrow & \bar{c}_{\mu}-\frac{3 \xi+4}{32} h \bar{c}_{\mu}-\frac{\xi+2}{8} h_{\mu}{ }^{\nu} \bar{c}_{\nu}+\frac{1}{\square}\left[-\frac{2 \xi^{2}-3 \xi+4}{16 \xi} \partial_{\rho} \partial_{\mu} h \bar{c}^{\rho}-\frac{\xi-1}{4 \xi} \partial_{\mu} \partial^{\sigma} h_{\sigma \rho} \bar{c}^{\rho}\right. \\
& \left.+\frac{1}{4 \xi} \partial_{\sigma} \partial_{\rho} h_{\mu}^{\sigma} \bar{c}^{\rho}-\frac{1+2 \xi}{4 \xi} \partial^{\rho} \partial^{\sigma} h_{\rho \sigma} \bar{c}_{\mu}-\frac{3 \xi^{2}-12 \xi-4}{32 \xi} \square h \bar{c}_{\mu}+\frac{1+2 \xi}{16} h \partial_{\mu} \partial_{\rho} \bar{c}^{\rho}\right] \\
h_{\mu \nu} \rightarrow & h_{\mu \nu}-\frac{1}{2} \bar{c}_{(\mu} c_{\nu)}
\end{aligned}
$$

Although the above is non-local, of course, the resulting action is local. This follows from the fact that the linear component of the gauge-fixing functional was determined by the double-copy to be de Donder (2.27). This yields a specific form for the quadratic ghost action (2.28) proportional to $\bar{c}_{\rho} \square c^{\rho}$, prior to any field redefinitions, which excludes all possibly non-local terms that may have arisen from (3.21).

\footnotetext{
${ }^{6}$ Recall, we are setting the dilaton in Einstein-frame to vanish, and thus, forbidding further field redefinitions.
} 


\section{Conclusions}

In this paper, we demonstrated that the BRST convolution product, in conjunction with the BCJ algorithm, can reproduce the Lagrangian of pure BRST Einstein-Hilbert gravity up to cubic order. We found that the ghost sector of the Yang-Mills action played a crucial rôle in achieving this in the pure graviton sector. We additionally derived the gauge fixing functional up to second order in fluctuations and the corresponding diffeomorphism ghost action.

We have focused here on the pure gravity case as a proof of principle, in particular that the graviton and dilaton may be disentangled from one-another. While the inclusion of the full $\mathcal{N}=0$ multiplet would in fact simplify the analysis in certain regards, as reflected by the double-copy amplitudes, it is not required for this purpose and, moreover, would serve to obscure this point. However, the full $\mathcal{N}=0$ supergravity construction, including the two-form and dilaton, would be of interest, both conceptually and from the perspective of classical solutions $[6,69,96,127]$. Work on this is in progress [126].

Another obvious generalisation would be to promote one of the factors to a full offshell $\mathcal{N}=1$ vector supermultiplet as in [1]. In this case, the ghost-antoghost sector would produce an entire chiral multiplet, reflecting the fact that on-shell " $\mathcal{N}=0$ Yang-Mills $\times$ $\mathcal{N}=1$ Yang-Mills" yields $\mathcal{N}=1$ supergravity coupled to a chiral multiplet.

We also note that we have made a choice of a linear gauge fixing functional $G[A]^{a}=$ $\partial^{\rho} A_{\rho}^{a}$ for the YM theory. It would very instructive to study non-linear gauge choices - the challenge in this context would be to understand how the BCJ rules need to be modified.

Note, we have from the beginning eliminated the Nakanishi-Lautrup auxiliary field corresponding to the Yang-Mills antighost. It would perhaps be instructive to understand what rôle it might play in the convolution product. The full Batalin-Vilkovisky formalism and BRST complex will be treated, from an independent perspective not relying on the convolution product, in work in progress [128].

Of course, an important question is how to proceed to higher orders in perturbation theory. A path towards this would possibly make use of the BCJ respecting Yang-Mills Lagrangians of [5] and [59], which include identically vanishing non-local terms to all orders that then need to be made local and cubic through the introduction of auxiliary fields as described at four and five points in [5].

\section{Acknowledgments}

We are grateful to Leonardo de la Cruz, Donal O'Connell, Andres Luna, Ricardo Monteiro and Chris White for helpful discussions. LB is particularly grateful to Hyungrok Kim for illuminating conversations. SN is supported by STFC grant ST/P000703/1 and a Leverhulme Research Project Grant. The work of LB has been supported by a Schrödinger Fellowship and the Leverhulme Trust.

Open Access. This article is distributed under the terms of the Creative Commons Attribution License (CC-BY 4.0), which permits any use, distribution and reproduction in any medium, provided the original author(s) and source are credited. 


\section{References}

[1] A. Anastasiou, L. Borsten, M.J. Duff, L.J. Hughes and S. Nagy, Yang-Mills origin of gravitational symmetries, Phys. Rev. Lett. 113 (2014) 231606 [arXiv:1408.4434] [InSPIRE].

[2] A. Anastasiou, L. Borsten, M.J. Duff, S. Nagy and M. Zoccali, Gravity as Gauge Theory Squared: A Ghost Story, Phys. Rev. Lett. 121 (2018) 211601 [arXiv: 1807.02486] [InSPIRE].

[3] Z. Bern, J.J.M. Carrasco and H. Johansson, New Relations for Gauge-Theory Amplitudes, Phys. Rev. D 78 (2008) 085011 [arXiv:0805.3993] [InSPIRE].

[4] Z. Bern, J.J.M. Carrasco and H. Johansson, Perturbative Quantum Gravity as a Double Copy of Gauge Theory, Phys. Rev. Lett. 105 (2010) 061602 [arXiv:1004.0476] [InSPIRE].

[5] Z. Bern, T. Dennen, Y.-t. Huang and M. Kiermaier, Gravity as the Square of Gauge Theory, Phys. Rev. D 82 (2010) 065003 [arXiv: 1004.0693] [INSPIRE].

[6] A. Luna et al., Perturbative spacetimes from Yang-Mills theory, JHEP 04 (2017) 069 [arXiv: 1611.07508] [INSPIRE].

[7] H. Kawai, D.C. Lewellen and S.H.H. Tye, A Relation Between Tree Amplitudes of Closed and Open Strings, Nucl. Phys. B 269 (1986) 1 [INSPIRE].

[8] M. Kiermaier, Gravity as the square of gauge theory, proceedings of Amplitudes 2010, Queen Mary, University of London, London U.K. 2010.

[9] N.E.J. Bjerrum-Bohr, P.H. Damgaard, T. Sondergaard and P. Vanhove, The Momentum Kernel of Gauge and Gravity Theories, JHEP 01 (2011) 001 [arXiv:1010.3933] [INSPIRE].

[10] C.R. Mafra, O. Schlotterer and S. Stieberger, Explicit BCJ Numerators from Pure Spinors, JHEP 07 (2011) 092 [arXiv:1104.5224] [INSPIRE].

[11] Y.-J. Du and C.-H. Fu, Explicit BCJ numerators of nonlinear simga model, JHEP 09 (2016) 174 [arXiv: 1606. 05846] [INSPIRE].

[12] Z. Bern, J.J. Carrasco, L.J. Dixon, H. Johansson and R. Roiban, The Ultraviolet Behavior of $N=8$ Supergravity at Four Loops, Phys. Rev. Lett. 103 (2009) 081301 [arXiv: 0905.2326] [INSPIRE].

[13] M. Chiodaroli, M. Günaydin and R. Roiban, Superconformal symmetry and maximal supergravity in various dimensions, JHEP 03 (2012) 093 [arXiv:1108.3085] [INSPIRE].

[14] Z. Bern, S. Davies, T. Dennen and Y.-t. Huang, Ultraviolet Cancellations in Half-Maximal Supergravity as a Consequence of the Double-Copy Structure, Phys. Rev. D 86 (2012) 105014 [arXiv: 1209.2472] [INSPIRE].

[15] J.J.M. Carrasco, M. Chiodaroli, M. Günaydin and R. Roiban, One-loop four-point amplitudes in pure and matter-coupled $\mathcal{N} \leq 4$ supergravity, JHEP 03 (2013) 056 [arXiv: 1212.1146] [INSPIRE].

[16] P.H. Damgaard, R. Huang, T. Sondergaard and Y. Zhang, The Complete KLT-Map Between Gravity and Gauge Theories, JHEP 08 (2012) 101 [arXiv:1206.1577] [INSPIRE].

[17] Y.-t. Huang and H. Johansson, Equivalent D $=3$ Supergravity Amplitudes from Double Copies of Three-Algebra and Two-Algebra Gauge Theories, Phys. Rev. Lett. 110 (2013) 171601 [arXiv: 1210.2255] [INSPIRE].

[18] T. Bargheer, S. He and T. McLoughlin, New Relations for Three-Dimensional Supersymmetric Scattering Amplitudes, Phys. Rev. Lett. 108 (2012) 231601 [arXiv: 1203.0562] [INSPIRE]. 
[19] J.J.M. Carrasco, R. Kallosh, R. Roiban and A.A. Tseytlin, On the U(1) duality anomaly and the $S$-matrix of $N=4$ supergravity, JHEP 07 (2013) 029 [arXiv:1303.6219] [InSPIRE].

[20] M. Chiodaroli, Q. Jin and R. Roiban, Color/kinematics duality for general abelian orbifolds of $N=4$ super Yang-Mills theory, JHEP 01 (2014) 152 [arXiv:1311.3600] [INSPIRE].

[21] H. Johansson and A. Ochirov, Pure Gravities via Color-Kinematics Duality for Fundamental Matter, JHEP 11 (2015) 046 [arXiv:1407.4772] [INSPIRE].

[22] M. Chiodaroli, M. Günaydin, H. Johansson and R. Roiban, Scattering amplitudes in $\mathcal{N}=2$ Maxwell-Einstein and Yang-Mills/Einstein supergravity, JHEP 01 (2015) 081 [arXiv: 1408.0764] [INSPIRE].

[23] M. Chiodaroli, M. Günaydin, H. Johansson and R. Roiban, Spontaneously Broken Yang-Mills-Einstein Supergravities as Double Copies, JHEP 06 (2017) 064 [arXiv:1511.01740] [INSPIRE].

[24] M. Chiodaroli, M. Günaydin, H. Johansson and R. Roiban, Complete construction of magical, symmetric and homogeneous $N=2$ supergravities as double copies of gauge theories, Phys. Rev. Lett. 117 (2016) 011603 [arXiv:1512.09130] [InSPIRE].

[25] M. Chiodaroli, Simplifying amplitudes in Maxwell-Einstein and Yang-Mills-Einstein supergravities, arXiv:1607.04129 [INSPIRE].

[26] J.J.M. Carrasco, C.R. Mafra and O. Schlotterer, Semi-abelian Z-theory: $N L S M \phi+{ }^{3}$ from the open string, JHEP 08 (2017) 135 [arXiv: 1612.06446] [INSPIRE].

[27] J.J.M. Carrasco, C.R. Mafra and O. Schlotterer, Abelian Z-theory: NLSM amplitudes and $\alpha$ '-corrections from the open string, JHEP 06 (2017) 093 [arXiv: 1608.02569] [INSPIRE].

[28] A. Anastasiou et al., Twin supergravities from Yang-Mills theory squared, Phys. Rev. D 96 (2017) 026013 [arXiv: 1610.07192] [INSPIRE].

[29] H. Johansson, G. Kälin and G. Mogull, Two-loop supersymmetric QCD and half-maximal supergravity amplitudes, JHEP 09 (2017) 019 [arXiv: 1706.09381] [INSPIRE].

[30] H. Johansson and J. Nohle, Conformal Gravity from Gauge Theory, arXiv:1707.02965 [INSPIRE].

[31] T. Azevedo and O.T. Engelund, Ambitwistor formulations of $R^{2}$ gravity and $(D F)^{2}$ gauge theories, JHEP 11 (2017) 052 [arXiv:1707.02192] [INSPIRE].

[32] A. Anastasiou, L. Borsten, M.J. Duff, A. Marrani, S. Nagy and M. Zoccali, Are all supergravity theories Yang-Mills squared?, Nucl. Phys. B 934 (2018) 606 [arXiv: 1707.03234] [INSPIRE].

[33] M. Chiodaroli, M. Günaydin, H. Johansson and R. Roiban, Explicit Formulae for Yang-Mills-Einstein Amplitudes from the Double Copy, JHEP 07 (2017) 002 [arXiv: 1703.00421] [INSPIRE].

[34] M. Chiodaroli, M. Günaydin, H. Johansson and R. Roiban, Gauged Supergravities and Spontaneous Supersymmetry Breaking from the Double Copy Construction, Phys. Rev. Lett. 120 (2018) 171601 [arXiv:1710.08796] [INSPIRE].

[35] M. Chiodaroli, M. Günaydin, H. Johansson and R. Roiban, Non-Abelian gauged supergravities as double copies, JHEP 06 (2019) 099 [arXiv: 1812.10434] [INSPIRE].

[36] Z. Bern, J.J.M. Carrasco, W.-M. Chen, H. Johansson, R. Roiban and M. Zeng, Five-loop four-point integrand of $N=8$ supergravity as a generalized double copy, Phys. Rev. D 96 (2017) 126012 [arXiv: 1708.06807] [INSPIRE]. 
[37] T. Azevedo, M. Chiodaroli, H. Johansson and O. Schlotterer, Heterotic and bosonic string amplitudes via field theory, JHEP 10 (2018) 012 [arXiv:1803.05452] [INSPIRE].

[38] A. Momeni, J. Rumbutis and A.J. Tolley, Massive Gravity from Double Copy, arXiv: 2004.07853 [INSPIRE].

[39] Z. Bern, J.J.M. Carrasco, L.J. Dixon, H. Johansson and R. Roiban, The Complete Four-Loop Four-Point Amplitude in $N=4$ Super-Yang-Mills Theory, Phys. Rev. D 82 (2010) 125040 [arXiv:1008.3327] [INSPIRE].

[40] J.J.M. Carrasco and H. Johansson, Five-Point Amplitudes in $N=4$ Super-Yang-Mills Theory and $N=8$ Supergravity, Phys. Rev. D 85 (2012) 025006 [arXiv:1106.4711] [INSPIRE].

[41] Z. Bern, C. Boucher-Veronneau and H. Johansson, $N \geq 4$ Supergravity Amplitudes from Gauge Theory at One Loop, Phys. Rev. D 84 (2011) 105035 [arXiv:1107.1935] [InSPIRE].

[42] C. Boucher-Veronneau and L.J. Dixon, $\mathcal{N} \geq 4$ Supergravity Amplitudes from Gauge Theory at Two Loops, JHEP 12 (2011) 046 [arXiv:1110.1132] [INSPIRE].

[43] Z. Bern, S. Davies, T. Dennen and Y.-t. Huang, Absence of Three-Loop Four-Point Divergences in $N=4$ Supergravity, Phys. Rev. Lett. 108 (2012) 201301 [arXiv:1202.3423] [INSPIRE].

[44] S. Oxburgh and C.D. White, BCJ duality and the double copy in the soft limit, JHEP 02 (2013) 127 [arXiv: 1210.1110] [INSPIRE].

[45] Z. Bern, J.J.M. Carrasco, L.J. Dixon, H. Johansson and R. Roiban, Simplifying Multiloop Integrands and Ultraviolet Divergences of Gauge Theory and Gravity Amplitudes, Phys. Rev. D 85 (2012) 105014 [arXiv:1201.5366] [INSPIRE].

[46] Y.-J. Du and H. Lüo, On General BCJ Relation at One-loop Level in Yang-Mills Theory, JHEP 01 (2013) 129 [arXiv:1207.4549] [INSPIRE].

[47] E.Y. Yuan, Virtual Color-Kinematics Duality: 6-pt 1-Loop MHV Amplitudes, JHEP 05 (2013) 070 [arXiv: 1210.1816] [InSPIRE].

[48] Z. Bern, S. Davies, T. Dennen, A.V. Smirnov and V.A. Smirnov, Ultraviolet Properties of $N=4$ Supergravity at Four Loops, Phys. Rev. Lett. 111 (2013) 231302 [arXiv:1309.2498] [INSPIRE].

[49] R.H. Boels, R.S. Isermann, R. Monteiro and D. O'Connell, Colour-Kinematics Duality for One-Loop Rational Amplitudes, JHEP 04 (2013) 107 [arXiv: 1301.4165] [INSPIRE].

[50] Z. Bern, S. Davies, T. Dennen, Y.-t. Huang and J. Nohle, Color-Kinematics Duality for Pure Yang-Mills and Gravity at One and Two Loops, Phys. Rev. D 92 (2015) 045041 [arXiv: 1303.6605] [INSPIRE].

[51] Z. Bern, S. Davies and T. Dennen, The Ultraviolet Structure of Half-Maximal Supergravity with Matter Multiplets at Two and Three Loops, Phys. Rev. D 88 (2013) 065007 [arXiv: 1305.4876] [INSPIRE].

[52] Z. Bern, S. Davies and T. Dennen, The Ultraviolet Critical Dimension of Half-Maximal Supergravity at Three Loops, arXiv:1412.2441 [INSPIRE].

[53] Z. Bern, S. Davies and T. Dennen, Enhanced ultraviolet cancellations in $\mathcal{N}=5$ supergravity at four loops, Phys. Rev. D 90 (2014) 105011 [arXiv: 1409.3089] [INSPIRE].

[54] C.R. Mafra and O. Schlotterer, Two-loop five-point amplitudes of super Yang-Mills and supergravity in pure spinor superspace, JHEP 10 (2015) 124 [arXiv:1505.02746] [INSPIRE]. 
[55] Z. Bern et al., Ultraviolet Properties of $\mathcal{N}=8$ Supergravity at Five Loops, Phys. Rev. D 98 (2018) 086021 [arXiv: 1804.09311] [inSPIRE].

[56] Z. Bern, C. Cheung, H.-H. Chi, S. Davies, L. Dixon and J. Nohle, Evanescent Effects Can Alter Ultraviolet Divergences in Quantum Gravity without Physical Consequences, Phys. Rev. Lett. 115 (2015) 211301 [arXiv:1507.06118] [INSPIRE].

[57] R. Monteiro and D. O'Connell, The Kinematic Algebra From the Self-Dual Sector, JHEP 07 (2011) 007 [arXiv: 1105.2565] [INSPIRE].

[58] N.E.J. Bjerrum-Bohr, P.H. Damgaard, R. Monteiro and D. O'Connell, Algebras for Amplitudes, JHEP 06 (2012) 061 [arXiv:1203.0944] [INSPIRE].

[59] M. Tolotti and S. Weinzierl, Construction of an effective Yang-Mills Lagrangian with manifest BCJ duality, JHEP 07 (2013) 111 [arXiv:1306.2975] [INSPIRE].

[60] R. Monteiro and D. O'Connell, The Kinematic Algebras from the Scattering Equations, JHEP 03 (2014) 110 [arXiv:1311.1151] [InSPIRE].

[61] C.-H. Fu and K. Krasnov, Colour-Kinematics duality and the Drinfeld double of the Lie algebra of diffeomorphisms, JHEP 01 (2017) 075 [arXiv: 1603.02033] [INSPIRE].

[62] C. Cheung and C.-H. Shen, Symmetry for Flavor-Kinematics Duality from an Action, Phys. Rev. Lett. 118 (2017) 121601 [arXiv:1612.00868] [INSPIRE].

[63] G. Chen, H. Johansson, F. Teng and T. Wang, On the kinematic algebra for BCJ numerators beyond the MHV sector, JHEP 11 (2019) 055 [arXiv:1906.10683] [INSPIRE].

[64] R. Monteiro, D. O'Connell and C.D. White, Black holes and the double copy, JHEP 12 (2014) 056 [arXiv: 1410.0239] [INSPIRE].

[65] A. Luna, R. Monteiro, D. O'Connell and C.D. White, The classical double copy for Taub-NUT spacetime, Phys. Lett. B $\mathbf{7 5 0}$ (2015) 272 [arXiv:1507.01869] [INSPIRE].

[66] A.K. Ridgway and M.B. Wise, Static Spherically Symmetric Kerr-Schild Metrics and Implications for the Classical Double Copy, Phys. Rev. D 94 (2016) 044023 [arXiv: 1512.02243] [INSPIRE].

[67] A. Luna, R. Monteiro, I. Nicholson, D. O'Connell and C.D. White, The double copy: Bremsstrahlung and accelerating black holes, JHEP 06 (2016) 023 [arXiv:1603.05737] [INSPIRE].

[68] C.D. White, Exact solutions for the biadjoint scalar field, Phys. Lett. B 763 (2016) 365 [arXiv: 1606.04724] [INSPIRE].

[69] W.D. Goldberger and A.K. Ridgway, Radiation and the classical double copy for color charges, Phys. Rev. D 95 (2017) 125010 [arXiv:1611.03493] [InSPIRE].

[70] G.L. Cardoso, S. Nagy and S. Nampuri, A double copy for $\mathcal{N}=2$ supergravity: a linearised tale told on-shell, JHEP 10 (2016) 127 [arXiv:1609.05022] [INSPIRE].

[71] G. Cardoso, S. Nagy and S. Nampuri, Multi-centered $\mathcal{N}=2$ BPS black holes: a double copy description, JHEP 04 (2017) 037 [arXiv:1611.04409] [INSPIRE].

[72] A. Luna, S. Nagy and C. White, The convolutional double copy: a case study with a point, arXiv:2004.11254 [INSPIRE].

[73] W.D. Goldberger, S.G. Prabhu and J.O. Thompson, Classical gluon and graviton radiation from the bi-adjoint scalar double copy, Phys. Rev. D 96 (2017) 065009 [arXiv:1705.09263] [INSPIRE]. 
[74] G. Lopes Cardoso, G. Inverso, S. Nagy and S. Nampuri, Comments on the double copy construction for gravitational theories, PoS CORFU2017 (2018) 177 [arXiv:1803.07670] [INSPIRE].

[75] A. Luna, I. Nicholson, D. O'Connell and C.D. White, Inelastic Black Hole Scattering from Charged Scalar Amplitudes, JHEP 03 (2018) 044 [arXiv:1711.03901] [INSPIRE].

[76] N. Bahjat-Abbas, A. Luna and C.D. White, The Kerr-Schild double copy in curved spacetime, JHEP 12 (2017) 004 [arXiv:1710.01953] [INSPIRE].

[77] D.S. Berman, E. Chacón, A. Luna and C.D. White, The self-dual classical double copy and the Eguchi-Hanson instanton, JHEP 01 (2019) 107 [arXiv: 1809.04063] [INSPIRE].

[78] J. Plefka, J. Steinhoff and W. Wormsbecher, Effective action of dilaton gravity as the classical double copy of Yang-Mills theory, Phys. Rev. D 99 (2019) 024021 [arXiv: 1807.09859] [INSPIRE].

[79] N. Bahjat-Abbas, R. Stark-Muchão and C.D. White, Biadjoint wires, Phys. Lett. B 788 (2019) 274 [arXiv: 1810.08118] [INSPIRE].

[80] A. Luna, R. Monteiro, I. Nicholson and D. O'Connell, Type D Spacetimes and the Weyl Double Copy, Class. Quant. Grav. 36 (2019) 065003 [arXiv:1810.08183] [InSPIRE].

[81] C.-H. Shen, Gravitational Radiation from Color-Kinematics Duality, JHEP 11 (2018) 162 [arXiv: 1806.07388] [INSPIRE].

[82] C. Cheung, I.Z. Rothstein and M.P. Solon, From Scattering Amplitudes to Classical Potentials in the Post-Minkowskian Expansion, Phys. Rev. Lett. 121 (2018) 251101 [arXiv: 1808.02489] [INSPIRE].

[83] D.A. Kosower, B. Maybee and D. O'Connell, Amplitudes, Observables and Classical Scattering, JHEP 02 (2019) 137 [arXiv:1811.10950] [INSPIRE].

[84] M. Carrillo González, B. Melcher, K. Ratliff, S. Watson and C.D. White, The classical double copy in three spacetime dimensions, JHEP 07 (2019) 167 [arXiv:1904.11001] [INSPIRE].

[85] H. Johansson and A. Ochirov, Double copy for massive quantum particles with spin, JHEP 09 (2019) 040 [arXiv: 1906.12292] [INSPIRE].

[86] B. Maybee, D. O'Connell and J. Vines, Observables and amplitudes for spinning particles and black holes, JHEP 12 (2019) 156 [arXiv:1906.09260] [INSPIRE].

[87] J. Plefka, C. Shi, J. Steinhoff and T. Wang, Breakdown of the classical double copy for the effective action of dilaton-gravity at NNLO, Phys. Rev. D 100 (2019) 086006 [arXiv: 1906. 05875] [INSPIRE].

[88] Z. Bern, C. Cheung, R. Roiban, C.-H. Shen, M.P. Solon and M. Zeng, Scattering Amplitudes and the Conservative Hamiltonian for Binary Systems at Third Post-Minkowskian Order, Phys. Rev. Lett. 122 (2019) 201603 [arXiv:1901.04424] [INSPIRE].

[89] Z. Bern, C. Cheung, R. Roiban, C.-H. Shen, M.P. Solon and M. Zeng, Black Hole Binary Dynamics from the Double Copy and Effective Theory, JHEP 10 (2019) 206 [arXiv:1908.01493] [INSPIRE].

[90] N. Arkani-Hamed, Y.-t. Huang and D. O'Connell, Kerr black holes as elementary particles, JHEP 01 (2020) 046 [arXiv: 1906.10100] [INSPIRE].

[91] R. Alawadhi, D.S. Berman, B. Spence and D. Peinador Veiga, S-duality and the double copy, JHEP 03 (2020) 059 [arXiv: 1911.06797] [INSPIRE]. 
[92] I. Bah, R. Dempsey and P. Weck, Kerr-Schild Double Copy and Complex Worldlines, JHEP 02 (2020) 180 [arXiv: 1910.04197] [INSPIRE].

[93] J. Plefka, C. Shi and T. Wang, Double copy of massive scalar QCD, Phys. Rev. D 101 (2020) 066004 [arXiv: 1911.06785] [inSPIRE].

[94] A. Banerjee, E. Ó Colgáin, J.A. Rosabal and H. Yavartanoo, Ehlers as EM duality in the double copy, arXiv:1912.02597 [INSPIRE].

[95] L. Alfonsi, C.D. White and S. Wikeley, Topology and Wilson lines: global aspects of the double copy, arXiv:2004.07181 [INSPIRE].

[96] K. Kim, K. Lee, R. Monteiro, I. Nicholson and D. Peinador Veiga, The Classical Double Copy of a Point Charge, JHEP 02 (2020) 046 [arXiv: 1912.02177] [InSPIRE].

[97] N. Bahjat-Abbas, R. Stark-Muchão and C.D. White, Monopoles, shockwaves and the classical double copy, JHEP 04 (2020) 102 [arXiv: 2001.09918] [INSPIRE].

[98] E. Lescano and J.A. Rodríguez, $\mathcal{N}=1$ Supersymmetric Double Field Theory and the generalized Kerr-Schild Ansatz, arXiv:2002.07751 [INSPIRE].

[99] Z. Bern, H. Ita, J. Parra-Martinez and M.S. Ruf, Universality in the classical limit of massless gravitational scattering, arXiv:2002.02459 [INSPIRE].

[100] J.J.M. Carrasco, Gauge and Gravity Amplitude Relations, in Journeys Through the Precision Frontier: Amplitudes for Colliders, World Scientific, (2015), DOI [arXiv: 1506.00974] [INSPIRE].

[101] Z. Bern, J.J. Carrasco, M. Chiodaroli, H. Johansson and R. Roiban, The Duality Between Color and Kinematics and its Applications, arXiv:1909.01358 [INSPIRE].

[102] L. Borsten, Gravity as the square of gauge theory: a review, Riv. Nuovo Cim. 43 (2020) 97.

[103] L. Borsten, M.J. Duff, L.J. Hughes and S. Nagy, Magic Square from Yang-Mills Squared, Phys. Rev. Lett. 112 (2014) 131601 [arXiv:1301.4176] [INSPIRE].

[104] L. Borsten, $D=6, \mathcal{N}=(2,0)$ and $\mathcal{N}=(4,0)$ theories, Phys. Rev. D 97 (2018) 066014 [arXiv: 1708.02573] [INSPIRE].

[105] L. Borsten, I. Jubb, V. Makwana and S. Nagy, Gauge $\times$ gauge on spheres, JHEP 06 (2020) 096 [arXiv: 1911.12324] [INSPIRE].

[106] A. Anastasiou, L. Borsten, M.J. Duff, L.J. Hughes and S. Nagy, A magic pyramid of supergravities, JHEP 04 (2014) 178 [arXiv:1312.6523] [INSPIRE].

[107] S. Nagy, Chiral Squaring, JHEP 07 (2016) 142 [arXiv:1412.4750] [INSPIRE].

[108] L. Borsten and M.J. Duff, Gravity as the square of Yang-Mills?, Phys. Scripta 90 (2015) 108012 [arXiv: 1602.08267] [INSPIRE].

[109] A. Anastasiou, L. Borsten, M.J. Hughes and S. Nagy, Global symmetries of Yang-Mills squared in various dimensions, JHEP 01 (2016) 148 [arXiv:1502.05359] [INSPIRE].

[110] L. Borsten, M.J. Duff and A. Marrani, Twin conformal field theories, JHEP 03 (2019) 112 [arXiv: 1812.11130] [INSPIRE].

[111] A. Hodges, New expressions for gravitational scattering amplitudes, JHEP 07 (2013) 075 [arXiv: 1108.2227] [INSPIRE].

[112] D. Vaman and Y.-P. Yao, Constraints and Generalized Gauge Transformations on Tree-Level Gluon and Graviton Amplitudes, JHEP 11 (2010) 028 [arXiv:1007.3475] [INSPIRE]. 
[113] F. Cachazo, S. He and E.Y. Yuan, Scattering of Massless Particles: Scalars, Gluons and Gravitons, JHEP 07 (2014) 033 [arXiv:1309.0885] [InSPIRE].

[114] F. Cachazo, S. He and E.Y. Yuan, Scattering Equations and Matrices: From Einstein To Yang-Mills, DBI and NLSM, JHEP 07 (2015) 149 [arXiv:1412.3479] [INSPIRE].

[115] S.G. Naculich, Scattering equations and BCJ relations for gauge and gravitational amplitudes with massive scalar particles, JHEP 09 (2014) 029 [arXiv:1407.7836] [INSPIRE].

[116] S.G. Naculich, CHY representations for gauge theory and gravity amplitudes with up to three massive particles, JHEP 05 (2015) 050 [arXiv:1501.03500] [INSPIRE].

[117] P.-J. De Smet and C.D. White, Extended solutions for the biadjoint scalar field, Phys. Lett. $B \mathbf{7 7 5}$ (2017) 163 [arXiv:1708.01103] [INSPIRE].

[118] R.W. Brown and S.G. Naculich, KLT-type relations for QCD and bicolor amplitudes from color-factor symmetry, JHEP 03 (2018) 057 [arXiv: 1802.01620] [INSPIRE].

[119] W. Siegel, Superstrings Give Old Minimal Supergravity, Phys. Lett. B 211 (1988) 55 [INSPIRE].

[120] W. Siegel, Curved extended superspace from Yang-Mills theory a la strings, Phys. Rev. D 53 (1996) 3324 [hep-th/9510150] [INSPIRE].

[121] L. Borsten and S. Nagy, Perturbative gravity from BRST squared, to appear.

[122] T. Kugo and I. Ojima, Local Covariant Operator Formalism of Nonabelian Gauge Theories and Quark Confinement Problem, Prog. Theor. Phys. Suppl. 66 (1979) 1 [InSPIRE].

[123] M. Henneaux and C. Teitelboim, Quantization of gauge systems Princeton University Press, Princeton U.S.A. (1992) [INSPIRE].

[124] J. Gomis, J. Paris and S. Samuel, Antibracket, antifields and gauge theory quantization, Phys. Rept. 259 (1995) 1 [hep-th/9412228] [INSPIRE].

[125] M. Zoccali, Supergravity as Yang-Mills Squared, Ph.D. thesis, Imperial College, London U.K. (2018).

[126] S. Nagy, $\mathcal{N}=0$ Supergravity to cubic order from BRST squared, in progress.

[127] W.D. Goldberger, J. Li and S.G. Prabhu, Spinning particles, axion radiation and the classical double copy, Phys. Rev. D 97 (2018) 105018 [arXiv:1712.09250] [INSPIRE].

[128] L. Borsten, H. Kim, B. Jurčo, T. Macrelli, C. Sämann and M. Wolf, Double-copy from homotopy algebras, to appear. 